\title{
Combining site and regional flood information using a Bayesian Monte Carlo approach
}

\author{
Tom Micevski ${ }^{1}$ and George Kuczera ${ }^{1}$ \\ Received 20 May 2008; revised 14 December 2008; accepted 12 January 2009; published 4 April 2009.
}

[1] Regression-based methods are widely used in flood regionalization. Since the generalized least squares (GLS) regression model properly accounts for both model and sampling errors, realistic estimates of uncertainty can be obtained. This study presents a general Bayesian approach for inferring the GLS regional regression model and for combining with any available site information to obtain the most accurate flood quantiles. A robust block Metropolis scheme is developed to sample the posterior distribution of the GLS regression model parameters. A particular feature of the sampler is the use of a uniform proposal for the regional model error variance to avoid convergence difficulties. A simple but general procedure, based on importance sampling, is developed to combine any kind of site information with regional information. The benefit of this approach is that all available information is fully exploited, and uncertainty is rigorously quantified as well as being minimized. Two case studies are presented. A synthetic case study illustrates the complex nature of the posterior distribution for the regional model error variance with its shape dependent on how much sampling error dominates the total error in the regional GLS model. A second case study involving 24 sites from the east coast of Australia is presented for the log Pearson III model. It demonstrates the significant benefit of combining site and regional information and the quantification of uncertainty.

Citation: Micevski, T., and G. Kuczera (2009), Combining site and regional flood information using a Bayesian Monte Carlo approach, Water Resour. Res., 45, W04405, doi:10.1029/2008WR007173.

\section{Introduction}

[2] In flood frequency analysis the objective is to infer the flood probability model described by the probability density function (pdf) $p(q \mid \theta)$ where $q$ is the annual maximum flood and $\theta$ is a vector of parameters characterizing the probability model. Information about $\theta$ can come from site information such as gauged flows and historic information, and from information at other catchments transferred to the site of interest using a regional model. The combining or pooling of both sources of information is advantageous in most cases because it leads to reduced uncertainty [Kuczera, 1983]. Perhaps the best known example is presented in Bulletin 17B [Interagency Advisory Committee on Water Data, 1982] which describes a variance-weighted procedure for pooling site and regional estimates of skewness. It should be noted that Bulletin $17 B$ is currently under review, with Griffis and Stedinger [2007a] providing a review of the history of the existing procedures and recommendations for future improvements.

[3] In view of the fact that practical application of flood frequency analysis is typically constrained by limited information, it is worthwhile revisiting the question of how to make the best use of the available information to estimate flood risk. Though it has been well recognized that the

\footnotetext{
${ }^{1}$ Discipline of Civil, Surveying, and Environmental Engineering, University of Newcastle, Callaghan, New South Wales, Australia.

Bayesian paradigm provides a very flexible framework to efficiently pool different kinds of information, its usage has been limited by implementation obstacles. Fortunately, with recent advances in Markov chain Monte Carlo methods to sample from multivariate probability distributions (for a recent treatment, see, e.g., Robert and Casella [2004]), the full potential of Bayesian methods is now more accessible.

[4] The principal contribution of this study is to reassess the Bayesian framework for pooling site and regional information using Monte Carlo methods. The widely used regional linear model is considered. A robust two-step block Metropolis scheme is outlined to evaluate the posterior distribution of the regional model parameters. Its output is in the form of a large sample of regional model parameters. A simple and general procedure is presented to utilize this regional information, expressed in the form of a random sample from the regional posterior distribution, with any available site information to produce a pooled flood frequency inference. Two case studies, one synthetic and one actual, illustrate the method.

\section{Regional Regression Model}

[5] Regression-based methods are widely used in flood regionalization. These methods map a set of catchment descriptor variables into a set of hydrologic variables. Typically, the relationship is assumed to be a linear function. For the $i$ th site, it is

$$
\theta_{i}=z_{i 1} \beta_{1}+z_{i 2} \beta_{2}+\cdots+z_{i m} \beta_{m}+\delta_{i}
$$


where $\theta_{i}$ is a scalar variable of interest (e.g., a parameter from the flood probability model or a flood quantile), $z_{i j}$ is the $j$ th catchment descriptor (e.g., catchment area, channel slope), $\beta_{j}$ is the $j$ th regional regression parameter, and $\delta_{i}$ is the regional model error. For $n$ sites and $m$ catchment descriptors, this relationship can be rewritten in matrix form:

$$
\Theta=Z \beta+\delta
$$

where $\Theta=\left[\theta_{1}, \ldots, \theta_{n}\right]^{T}, Z$ is an $n \times m$ matrix, $\beta=\left[\beta_{1}, \ldots\right.$, $\left.\beta_{m}\right]^{T}$, and $\delta=\left[\delta_{1}, \ldots, \delta_{n}\right]^{T}$. In general, the vector of regional model errors is assumed to have zero mean and a covariance matrix of the form

$$
\operatorname{Cov}(\delta)=\sigma_{R}^{2} V
$$

where $\sigma_{R}^{2}$ is the regional model error variance and $V$ is a symmetric, positive definite matrix [Theil, 1971].

[6] Different assumptions about the structure of the model errors can be made by the appropriate selection of the matrix $V$. A traditional approach often used in hydrology is the ordinary least squares (OLS) method, which assumes that the model errors are independently distributed with zero mean and a common variance $\sigma_{R}^{2}$; that is,

$$
\operatorname{Cov}(\delta)=\sigma_{R}^{2} I
$$

where $I$ is an $n \times n$ identity matrix. This approach is appropriate when the $\theta_{i}$ are estimated with negligible sampling error and there is no spatial correlation in the regional model errors between sites. However, in practice, sampling error cannot be neglected [Tasker and Stedinger, 1989].

[7] Equation (1) assumes that the true value of $\theta$ is available. However, typically only estimates of $\theta$ are available and are denoted by $\theta$. As a result, sampling error associated with the estimation of $\theta$ needs to be filtered out in order to correctly infer the model error variance $\sigma_{R}^{2}$ and the regional regression parameters $\beta$ [Kuczera, 1983]. To accomplish this, Stedinger and Tasker [1985, 1986a, 1986b] posed the regional model as a generalized least squares (GLS) model

$$
\hat{\Theta}=Z \beta+\delta+\varepsilon=Z \beta+\eta
$$

where $\hat{\Theta}$ is a vector of estimates of $\theta, \varepsilon$ is a time-sampling error vector arising from the fact that $\theta$ is estimated from a limited flood record. The total regression error $\eta$ has the properties $\mathrm{E}(\eta)=0$ and covariance

$$
\operatorname{Cov}(\eta)=\Lambda\left(\sigma_{R}^{2}\right)=\sigma_{R}^{2} I+\Sigma
$$

where $\Sigma$ is the covariance matrix of time-sampling errors, which are usually correlated. Estimation of this covariance matrix is an important task because it describes the precision of the estimator $\hat{\theta}$. The structure and form of the sampling covariance matrix $\Sigma$ varies with both the variable of interest $\theta$ and the underlying flood distribution.

[8] In a regional flood frequency study, a key step is to infer the regional model parameters $\beta$ and $\sigma_{R}^{2}$. The inference of the regional model error variance $\sigma_{R}^{2}$ is particularly important as $\sigma_{R}^{2}$ expresses the noise in the regional relationship between site variables and descriptors. Both Kuczera [1983] and Stedinger and Tasker [1985] show that sampling theory estimates of $\sigma_{R}^{2}$ can produce negative values. In such cases, Stedinger and Tasker [1985] suggested setting $\sigma_{R}^{2}$ to zero, a not altogether satisfactory resolution of the problem. Recently Reis et al. [2005] derived a "quasi-analytical" Bayesian solution to overcome this problem.

\section{Regional Model Calibration Using the Block Metropolis Sampler}

[9] As in work by Reis et al. [2005], we adopt a Bayesian approach to infer the regional model parameters $\beta$ and $\sigma_{R}^{2}$. However, rather than employing quasi-analytic expressions, we develop a simple and robust Monte Carlo approach to sample from the posterior distribution of the regional parameters $\sigma_{R}^{2}$ and $\beta$. The key advantage of our approach is that it leads to a simple and general Monte Carlo algorithm for inferring the regional model parameters and for making predictions at a site using regional and any available site information for any type of flood probability model.

\subsection{Overview of Bayesian Method}

[10] The regional model parameters $\beta$ and $\sigma_{R}^{2}$ are estimated using Bayesian inference, which uses a probability distribution to describe what is known about the true value of the model parameters given the available information. Central to the Bayesian approach is Bayes theorem:

$$
p(\Phi \mid Y)=f(Y \mid \Phi) p(\Phi) / p(Y) \propto f(Y \mid \Phi) p(\Phi)
$$

where $p(\Phi \mid Y)$ is the posterior density describing the current knowledge about the model parameter vector $\Phi$ given the observed data $Y, f(Y \mid \Phi)$ is the likelihood function that defines the sampling distribution of $Y$ for a particular $\Phi$ and can be interpreted as the goodness-of-fit function, $p(\Phi)$ is the prior density that expresses the analyst's prior knowledge (which may be subjective) about the true value of $\Phi$, and $p(Y)$ is the marginal likelihood which plays the role of a normalizing constant.

[11] A major objective of Bayesian inference is evaluating and interpreting the posterior distribution. However, it is often not possible to derive an analytic expression, so other methods must be employed to explore the posterior distribution. The techniques often used are those known as Markov chain Monte Carlo (MCMC) methods [e.g., Gelman et al., 1995; Robert and Casella, 2004]. MCMC methods provide a general means to randomly sample from virtually any multivariate probability distribution, which, by design, is chosen to be the posterior distribution. Well-known implementations of MCMC methods include the MetropolisHastings algorithm [Metropolis et al., 1953; Hastings, 1970] and the Gibbs sampler [Geman and Geman, 1984], with Marshall et al. [2004] providing a comparison of the performance of several MCMC methods in a hydrological context.

[12] For the regional GLS regression model, a two-step block Metropolis sampler is used to draw samples from the posterior distributions of $\beta$ and $\sigma_{R}^{2}$. Before the two-step block Metropolis sampler procedure is described, the (single block) Metropolis-Hastings algorithm is first outlined. 


\subsection{Overview of the Metropolis-Hastings Algorithm}

[13] The Metropolis-Hastings algorithm is discussed in detail by Chib and Greenberg [1995]. The objective is to draw samples from the posterior distribution. For the $j$ th iteration of the Metropolis-Hastings sampler, a trial parameter $\Phi^{*}$ is sampled from a proposal distribution $J$ :

$$
\Phi^{*} \leftarrow J\left(\Phi^{*} \mid \Phi^{(j-1)}\right)
$$

where $\leftarrow$ denotes "is sampled from." While the selection of the proposal distribution is arbitrary, it is noted that a proposal that approximates the posterior distribution improves the performance and efficiency of the sampler. The trial parameter is then subjected to an acceptance/ rejection test:

$$
\Phi^{(j)}= \begin{cases}\Phi^{*} & \text { if } u \leq \min (r, 1) \\ \Phi^{(j-1)} & \text { otherwise }\end{cases}
$$

where $u$ is a random draw from the uniform distribution $\mathrm{U}(0,1)$ and

$$
r=\frac{p\left(\Phi^{*} \mid Y\right) / J\left(\Phi^{*} \mid \Phi^{(j-1)}\right)}{p\left(\Phi^{(j-1)} \mid Y\right) / J\left(\Phi^{(j-1)} \mid \Phi^{*}\right)}
$$

[14] If the proposal distribution is chosen to be symmetric, such that $J(A \mid B)=J(B \mid A), r$ reduces to the ratio of posterior densities:

$$
r_{\mathrm{MET}}=p\left(\Phi^{*} \mid Y\right) / p\left(\Phi^{(j-1)} \mid Y\right)
$$

This simplification is known as the Metropolis algorithm. Selection of the initial starting parameter value $\Phi^{(0)}$ is arbitrary; however, a poor initial selection may delay convergence to the target (i.e., posterior) distribution.

\subsection{Block Metropolis Sampler Implementation}

[15] The block Metropolis sampler is a generalization of the (single block) Metropolis-Hastings sampler where the Metropolis-Hastings algorithm is applied to subsets (blocks) of parameters, rather than simultaneously to the entire parameter vector. The full parameter vector for the regional model is first partitioned into two blocks $\Phi=\left\{\sigma_{R}^{2}, \beta\right\}$ to take advantage of the known solution for the conditional posterior of $\beta$. Each iteration of the block Metropolis sampler cycles through the two blocks of $\Phi$, randomly sampling each block from its conditional posterior distribution. The method is implemented using the following steps.

[16] Step 0 initializes the regional model parameter vector $\beta^{(0)}$ and the model error variance upper bound $\sigma_{\text {Max }}^{2}$. The $j$ th iteration of the block Metropolis sampler then proceeds as follows.

[17] Step 1 randomly samples a regional model variance $\sigma_{R}^{2}$ using a Metropolis-Hastings step: $\sigma_{R}^{2(j)} \leftarrow p\left(\sigma_{R}^{2} \mid \beta^{(j-1)}\right.$, $\hat{\Theta}, Z)$.

[18] Step 2 randomly samples a regional model parameter vector $\beta$ using a Gibbs step: $\beta^{(j)} \leftarrow p\left(\beta \mid \sigma_{R}^{2(j)}, \hat{\Theta}, Z\right)$.

[19] Steps $1-2$ are iterated $j=1, \ldots, N$ times. A detailed explanation of these steps follows.

3.3.1. Step 0: Initialization

[20] Before starting the block Metropolis sampler, OLS is used to provide an initial unbiased estimate for $\beta$, denoted by $\beta^{(0)}$. An upper bound on the model error variance is then estimated as [e.g., Theil, 1971, p. 114, equation (3.8)]

$$
\sigma_{\text {Max }}^{2}=\frac{K}{n-m}\left(\hat{\Theta}-Z \beta^{(0)}\right)^{T}\left(\hat{\Theta}-Z \beta^{(0)}\right)
$$

where $K$ is a constant, say in the range 2 to 4 . It is important to check that the posterior of $\sigma_{R}^{2}$ is not constrained by $K$. If it is, then $K$ would need to be increased.

\subsubsection{Step 1: Sample Regional Model Variance}

[21] Sample a trial model error variance from a uniform distribution with limits 0 and $\sigma_{\text {Max }}^{2}$; that is,

$$
\sigma_{R}^{2 *} \leftarrow \mathrm{U}\left(0, \sigma_{\text {Max }}^{2}\right)
$$

The choice of a uniform distribution was deliberate. On the basis of unpublished work, we have found the uniform distribution to be more robust than inverse-gamma-type distributions, particularly as $\sigma_{R}^{2} \rightarrow 0$. Because computer run times are of the order of a minute, we opted for a proposal that behaved robustly.

[22] Since the uniform proposal is symmetric, the Metropolis-Hastings acceptance ratio reduces to

$$
r=r_{\mathrm{MET}}=\frac{p\left(\sigma_{R}^{2 *} \mid \beta^{(j-1)}, \hat{\Theta}, Z\right)}{p\left(\sigma_{R}^{2(j-1)} \mid \beta^{(j-1)}, \hat{\Theta}, Z\right)}
$$

where

$$
p\left(\sigma_{R}^{2 *} \mid \beta^{(j-1)}, \hat{\Theta}, Z\right) \propto \mathrm{N}\left(\hat{\Theta} \mid Z \beta^{(j-1)}, \Sigma+\sigma_{R}^{2 *} I\right) p\left(\sigma_{R}^{2 *}\right)
$$

with $\mathrm{N}(x \mid m, S)$ denoting the multinormal pdf evaluated at vector $x$ with mean vector $m$ and covariance matrix $S$ and $p\left(\sigma_{R}^{2 *}\right)$ is the prior pdf, which for convenience can be set to $\mathrm{U}\left(0, \sigma_{\text {Max }}^{2}\right)$. The proposal is then subjected to an acceptance/ rejection step

$$
\sigma_{R}^{2(j)}= \begin{cases}\sigma_{R}^{2 *} & \text { if } u \leq r \\ \sigma_{R}^{2(j-1)} & \text { otherwise }\end{cases}
$$

\subsubsection{Step 2: Sample Regional Model Parameter Vector}

[23] For the linear regression model, the conditional posterior distribution takes a convenient form allowing direct sampling [Gelman et al., 1995, pp. 255, 235-237]. The new value of $\beta$ is directly sampled according to

$$
\beta^{(j)} \leftarrow p\left(\beta \mid \sigma_{R}^{2(j)}, \hat{\Theta}, Z\right)=\mathrm{N}(\mathrm{E}[\beta], \operatorname{Cov}[\beta])
$$

where

$$
\begin{gathered}
\mathrm{E}[\beta]=\left\{Z^{T}\left[\Sigma+\sigma_{R}^{2(j)} I\right]^{-1} Z\right\}^{-1} Z^{T}\left[\Sigma+\sigma_{R}^{2(j)} I\right]^{-1} \hat{\Theta} \\
\operatorname{Cov}[\beta]=\left\{Z^{T}\left[\Sigma+\sigma_{R}^{2(j)} I\right]^{-1} Z\right\}^{-1}
\end{gathered}
$$

As a concluding remark, the design of the algorithm has been guided by the need for robustness. The adoption of a 
uniform proposal in step 1 and sampling from a multivariate normal in step 2 maximize the chance of the algorithm successfully converging to the posterior. Note that the twostep block Metropolis procedure can be readily extended to a more complex model involving nonlinear dependency between site descriptors and model errors. In such cases, step 2 would require a Metropolis-Hastings step.

\section{Combining Site and Regional Information}

[24] We now consider the case of prediction at a site not used in developing the regional model. The regional model will be used as the prior and updated with any available site information to produce the posterior.

[25] Suppose there are $p$ variables of interest. For example, one may be interested in developing regional relationships for the three log Pearson III parameters which would lead to $p=3$ regional models. The output of the block Metropolis sampler for the $p$ regional models would be $\left\{\beta_{k}^{(j)}, \sigma_{k R}^{2}{ }^{(j)}, j=1, \ldots, N ; k=1, \ldots, p\right\}$. It is assumed here that the regional models are statistically independent of each other. The question arises: how can this sample be used in conjunction with any available site information to make a pooled inference about flood frequency quantities such as quantiles? The most direct approach would use the regional sample in the computation of the pooled posterior. This can be done using importance or particle sampling, an example of which was presented by Kuczera [1999].

\subsection{Importance Sampling}

[26] To motivate the procedure, Monte Carlo importance sampling is briefly outlined. The objective is to randomly sample from the (multivariate) pdf $f(x)$ denoted by

$$
x \leftarrow f(x)
$$

where $x$ is a $n$ vector. It is assumed there is no straightforward way to sample directly from $f(x)$. Importance sampling offers a simple and often satisfactory solution to this problem [see, e.g., Gelman et al., 1995; Robert and Casella, 2004]. It is based on the idea of sampling from a convenient proposal pdf $h(x)$ (which spans the domain of $f(x))$ and weighting the sample to compensate for the difference between $f(x)$ and $h(x)$.

[27] Consider the expected value of some function $g(x)$

$$
\mathrm{E}[g(x)]=\int g(x) f(x) d x
$$

This can be reexpressed as

$$
\mathrm{E}[g(x)]=\int g(x) \frac{f(x)}{h(x)} h(x) d x
$$

An approximation to this integral can be obtained using the method of Monte Carlo integration

$$
\begin{array}{rlrl}
\hat{\mathrm{E}}[g(x)] & =\frac{1}{N} \sum_{i=1}^{N} g\left(x_{i}\right) \frac{f\left(x_{i}\right)}{h\left(x_{i}\right)}, & & x_{i} \leftarrow h(x) \\
& =\sum_{i=1}^{N} g\left(x_{i}\right) \frac{1}{N} \frac{f\left(x_{i}\right)}{h\left(x_{i}\right)}, & x_{i} \leftarrow h(x) \\
& =\sum_{i=1}^{N} g\left(x_{i}\right) w\left(x_{i}\right), \quad x_{i} \leftarrow h(x)
\end{array}
$$

where the weight $w\left(x_{i}\right)$ can be interpreted as the probability mass assigned to the random sample (or "particle") $x_{i}$.

[28] The importance sampling algorithm yields $N$ particles from the distribution $f(x)$ with each particle having the (normalized) probability mass

$$
P\left(x_{i}\right)=\frac{\frac{f_{u}\left(x_{i}\right)}{h_{u}\left(x_{i}\right)}}{\sum_{k=1}^{N} \frac{f_{u}\left(x_{k}\right)}{h_{u}\left(x_{k}\right)}}, \quad i=1, \ldots, N
$$

where $f_{u}(x)$ and $h_{u}(x)$ denote the unnormalized pdfs of $f(x)$ and $h(x)$. One can randomly sample from the set of particles $\left\{x_{i}, P\left(x_{i}\right) ; i=1, \ldots, N\right\}$ to obtain a random sample from the distribution $f(x)$.

\subsection{Combining Site and Regional Information Using Importance Sampling}

[29] With the importance sampling algorithm established, the problem of combining site and regional information in a Monte Carlo framework can be resolved. At a particular site, the hydrologist is interested in making an inference about some function $g(\theta)$, where $\theta$ is the parameter vector characterizing the site flood frequency distribution $p(q \mid \theta)$. Typically the function $g(\theta)$ may be a particular flood quantile.

[30] The true value of the vector $\theta$ is unknown. However, the following information pertaining to $\theta$ is available to make an inference.

[31] 1. Using site descriptors denoted by the vector $z_{s}$, the $p$ regional models describe the probability distribution for $\theta$ in the form of a set of $p \times N$ samples

$$
\begin{aligned}
\theta_{k}^{(j)} & \leftarrow p\left(\theta_{k} \mid z_{s k}, R_{k}\right) \\
& =p\left(\theta_{k} \mid \beta_{k}^{(j)}, \sigma_{k R}^{(j)}, z_{s k}\right), \quad j=1, \ldots, N ; k=1, \ldots, p
\end{aligned}
$$

where $p\left(\theta_{k} \mid z_{s k}, R_{k}\right)$ denotes the regional posterior pdf of $\theta_{k}$ given the site characteristics $z_{s k}$ and the regional data $R_{k}=$ $\left\{\hat{\Theta}_{k}, Z_{k}\right\}$ used to calibrate the regional model.

[32] 2. Site-specific flood data $S$ relevant to the flood frequency distribution $p(q \mid \theta)$ is available. The data $S$ may consist of, for example, gauged peaks or pregauged information about historic peaks. The data enables the construction of the likelihood function $p(S \mid \theta)$ (see, e.g., Stedinger and Cohn [1986], Kuczera [1999], and Micevski et al. [2005] for details on how to compute the likelihood for different types of data).

[33] Both sources of information can be combined to obtain the Bayesian distribution of $g(\theta)$. The importance sampling algorithm described in the preceding section provides a framework for generating $N$ samples from the Bayesian distribution. Starting with the posterior expected value of $g(\theta)$

$$
\begin{aligned}
\mathrm{E}[g(\theta)] & =\int g(\theta) p\left(\theta \mid S, z_{s}, R\right) d \theta \\
& =\int g(\theta) \frac{p(S \mid \theta) p\left(\theta \mid z_{s}, R\right)}{p\left(S \mid z_{S}, R\right)} d \theta
\end{aligned}
$$

where $p\left(\theta \mid S, z_{s}, R\right)$ is the posterior pdf of $\theta$ after combining site and regional information. 
[34] This expectation can be approximated by sampling from the regional posterior $p\left(\theta \mid z_{s}, R\right)$

$$
\begin{aligned}
\hat{\mathrm{E}}[g(\theta)] & =\frac{1}{N} \sum_{j=1}^{N} g\left(\theta^{(j)}\right) \frac{p\left(S \mid \theta^{(j)}\right)}{p\left(S \mid z_{s}, R\right)}, \quad \theta^{(j)} \leftarrow p\left(\theta \mid z_{s}, R\right) \\
& =\sum_{j=1}^{N} g\left(\theta^{(j)}\right) w\left(\theta^{(j)}\right), \quad \theta^{(j)} \leftarrow p\left(\theta \mid z_{s}, R\right)
\end{aligned}
$$

which yields $N$ particles from the Bayesian distribution of $g(\theta)$ with particle weights

$$
P_{g}\left(\theta^{(j)}\right)=\frac{p\left(S \mid \theta^{(j)}\right)}{\sum_{k=1}^{N} p\left(S \mid \theta^{(k)}\right)}, \quad \theta^{(j)} \leftarrow p\left(\theta \mid z_{s}, R\right), j=1, \ldots, N
$$

[35] The importance sampling algorithm thus involves drawing samples from the regional model and assigning a weight proportional to the sample's likelihood. As the site information increases the particle weight will be concentrated around samples close to the most likely value. Conversely, with decreasing site information, the likelihood will flatten toward a uniform distribution. As a result, the weight assigned to each particle will tend to equality whereby the regional distribution completely informs the inference.

[36] Though this is a simple and general algorithm, its practical utility depends on how well the regional distribution $p\left(\theta \mid z_{s}, R\right)$ overlaps with the likelihood $p(S \mid \theta)$. One would expect some sites to be outliers from the perspective of the regional model. In such cases the overlap may be poor with a limited number of particles having significant probability mass. It is therefore important to check the distribution of particle weights to guard against such cases. Gelman et al. [1995, pp. 307-308] suggests checking histograms of the largest particles (or their logarithms) and ensuring that the largest particles are not too large relative to the others.

\section{Case Studies}

[37] Two case studies are presented to illustrate different aspects of the approach. The first considers synthetic data to illustrate the difficult case when sampling error dominates the total error. The second considers application to the northeastern region of New South Wales, Australia, to illustrate the pooling of site and regional information and the quantification of predictive uncertainty.

\subsection{Synthetic Example}

[38] As this study was motivated by the problem of negative sample estimates of the regional variance [Reis et $a l ., 2005]$, the focus of the example is the behavior of the posterior of $\sigma_{R}^{2}$ as the sampling error starts to dominate the total error. The example considers the regional estimation of a variable with "data" generated according to the following scheme.

[39] 1. At each of 80 sites, the true value of the variable is sampled from the true regional model $\theta_{i} \leftarrow \mathrm{N}\left(2+0.3 z_{i}, \sigma_{R}^{2}\right)$,
Table 1. Summary of Regional Inference for Three Synthetic Data Scenarios $^{\mathrm{a}}$

\begin{tabular}{lccc}
\hline & \multicolumn{3}{c}{ Data Scenario } \\
\cline { 2 - 4 } \multicolumn{1}{c}{ Variable } & Scenario 1 & Scenario 2 & Scenario 3 \\
\hline True value of $\sigma_{R}^{2}$ & 0.25 & 0.40 & 1.00 \\
Posterior mean of $\sigma_{R}^{2}$ & $0.160(0.235)$ & $0.362(0.322)$ & $1.12(0.40)$ \\
$\begin{array}{l}\text { OLS estimate of regional } \\
\quad \text { error variance }\end{array}$ & 1.97 & 2.13 & 2.78 \\
Acceptance rate for $\sigma_{R}^{2}$ & 8.2 & 16.1 & 21.2 \\
$\quad$ sample step (\%) & & & \\
Posterior mean of $\beta_{1}$ & $1.96(0.33)$ & $1.95(0.37)$ & $1.97(0.48)$ \\
Posterior mean of $\beta_{2}$ & $0.282(0.037)$ & $0.279(0.040)$ & $0.270(0.050)$ \\
OLS mean of $\beta_{1}$ & $2.14(0.51)$ & $2.17(0.56)$ & $2.26(0.77)$ \\
OLS mean of $\beta_{2}$ & $0.275(0.049)$ & $0.272(0.054)$ & $0.262(0.074)$ \\
\hline
\end{tabular}

${ }^{a}$ True values of $\beta_{1}$ and $\beta_{2}$ are 2.0 and 0.3 , respectively. Standard deviation is given in parentheses.

$i=1, \ldots, 80$, where $z$ is a single catchment descriptor ranging from 3.1 to 15 .

[40] 2. For each site, the estimate of the true value of the site variable was sampled independently according to $\hat{\theta}_{i} \leftarrow$ $\mathrm{N}\left(\theta_{i},\left(0.25 \theta_{i}\right)^{2}\right), i=1, \ldots, 80$. This means site estimates have a sampling coefficient of variation of $25 \%$.

[41] Three data scenarios were analyzed corresponding to the following regional error variances $\sigma_{R}^{2}: 0.25,0.40$ and 1.00. For each scenario the posterior was evaluated using $N=100000$ samples (with CPU times less than 2 seconds on a $2 \mathrm{GHz}$ Dell Latitude D610) and compared against ordinary least squares (OLS) regression estimates. Table 1 summarizes the results. Several points deserve comment.

[42] 1. As the true $\sigma_{R}^{2}$ decreases, the posterior coefficient of variation for $\sigma_{R}^{2}$ increases to values exceeding one indicating the sampling error noise is making it harder to infer the underlying true value of $\sigma_{R}^{2}$. Nonetheless, the posterior of $\sigma_{R}^{2}$ encloses the true value.

[43] 2. The OLS error variance grossly overestimates $\sigma_{R}^{2}$. This is to be expected given that the OLS regression is not made "aware" of the sampling errors affecting the site estimates.

[44] 3. The posterior and OLS estimates of $\beta$ are similar and consistent with the true values. The uncertainty in $\beta$ is quite small (as would be expected with 80 sites) with OLS yielding consistently greater standard deviations for $\beta$.

[45] These considerations are consistent with earlier findings [e.g., Stedinger and Tasker, 1985, 1986b; Tasker et al., 1987] that the main advantage of GLS over OLS is that it can filter out the effects of sampling error and arrive at a better estimate of $\sigma_{R}^{2}$. However, as already noted, GLS can produce negative sample estimates of $\sigma_{R}^{2}$. Setting such estimates to zero is unsatisfactory as it overinflates the precision of the regional model.

[46] The advantage of the Bayesian MCMC approach becomes evident upon considering Figure 1, which plots the posterior of $\sigma_{R}^{2}$ for the three scenarios. For scenario 3 (true $\left.\sigma_{R}^{2}=1\right), \sigma_{R}^{2}$ is of the same order as the sampling error. As a result, the posterior of $\sigma_{R}^{2}$ is unimodal and exhibits a $\chi^{2}$-type shape. However, for scenario 1 (true $\sigma_{R}^{2}=0.25$ ) the sampling error dominates the total error. As a result, the posterior of $\sigma_{R}^{2}$ exhibits a $\mathrm{J}$ shape with the mode at 0 but an expected value of 0.160 . Scenario 2 (true $\sigma_{R}^{2}=0.40$ ) represents a transitionary phase where sampling error is starting to dominate the total error, resulting in a bimodal density with the dominant mode at 0 . 


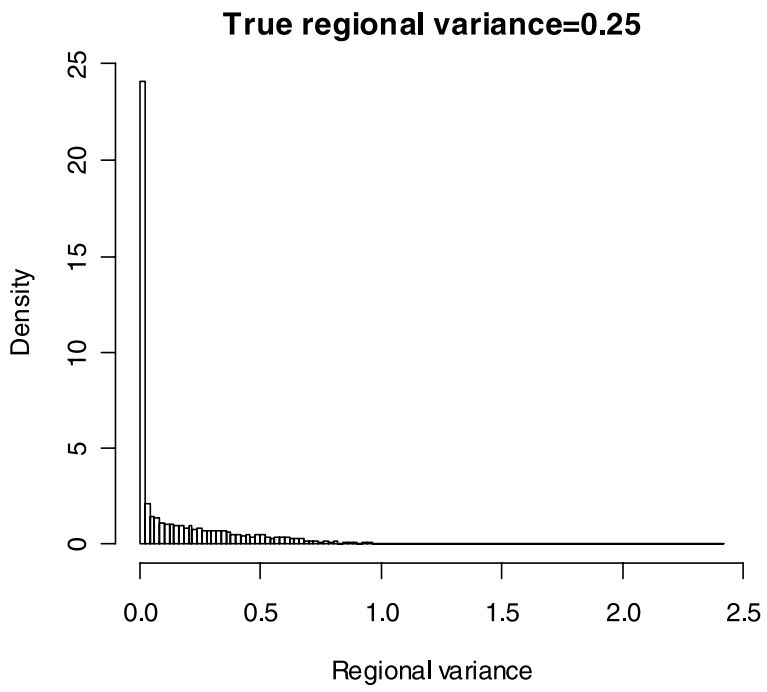

True regional variance $=0.4$

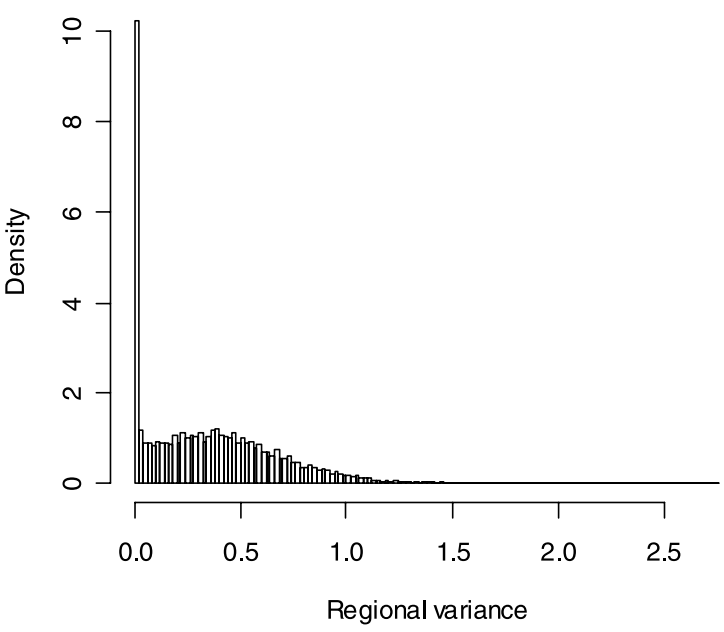

True regional variance $=1.0$

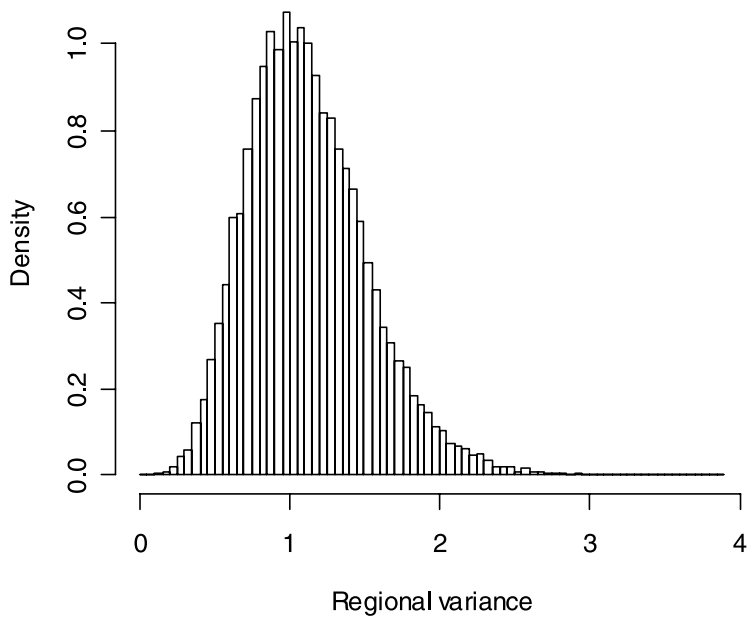

Figure 1. Posterior density of regional error variance $\sigma_{R}{ }^{2}$ with true value equal to (top to bottom) $0.25,0.4$, and 1.0 .

[47] We conclude that the posterior of $\sigma_{R}^{2}$ can exhibit quite complex behavior which classical approaches are unlikely to describe. Because the uncertainty in regional prediction is governed by $\sigma_{R}^{2}$, the case for a full Bayesian analysis is very strong.

\subsection{New South Wales Case Study}

[48] This case study illustrates application of the Bayesian regional GLS regression for the log Pearson III distribution applied to flood data in northeastern New South Wales (Australia) and demonstrates the combining or pooling of site and regional information. The distributions of selected quantiles are inferred using regional information alone and several combinations of gauged and regional information. The reductions in quantile uncertainty arising from pooling are demonstrated and highlight the importance of using, whenever possible, all valid sources of information.

\subsubsection{Flood Data}

[49] The flood data were obtained from the Pinneena database of the New South Wales (NSW) Department of Water and Energy. Instantaneous annual maximum discharges were extracted for 24 sites in northeastern NSW. The locations of these sites are shown in Figure 2. The region under study is large and ideally should be subdivided into smaller regions to enable stronger regional predictive performance. However, this is not a critical issue in this case study. The flood data are assumed to follow the widely used $\log$ Pearson III distribution with mean $\mu$, standard deviation $\sigma$, and skewness $\gamma$.

\subsubsection{Quantile Inference}

[50] For the sake of clarity, the importance samplingbased pooling procedure described in section 4 is presented in full detail.

[51] 1. The flood frequency distribution parameters are randomly sampled from their corresponding regional models. For the $j$ th regional parameter sample, we sample the three $\log$ Pearson parameters $\theta=\left\{\theta_{1}, \theta_{2}, \theta_{3}\right\}=\{\mu, \sigma, \gamma\}$ using

$$
\begin{aligned}
& \delta_{k}^{(j)} \leftarrow \mathrm{N}\left(0, \sigma_{k R}^{2(j)}\right) \\
& \theta_{k}^{(j)}=z_{s k}^{T} \beta_{k}^{(j)}+\delta_{k}^{(j)}
\end{aligned}
$$

for $k=1,2,3$.

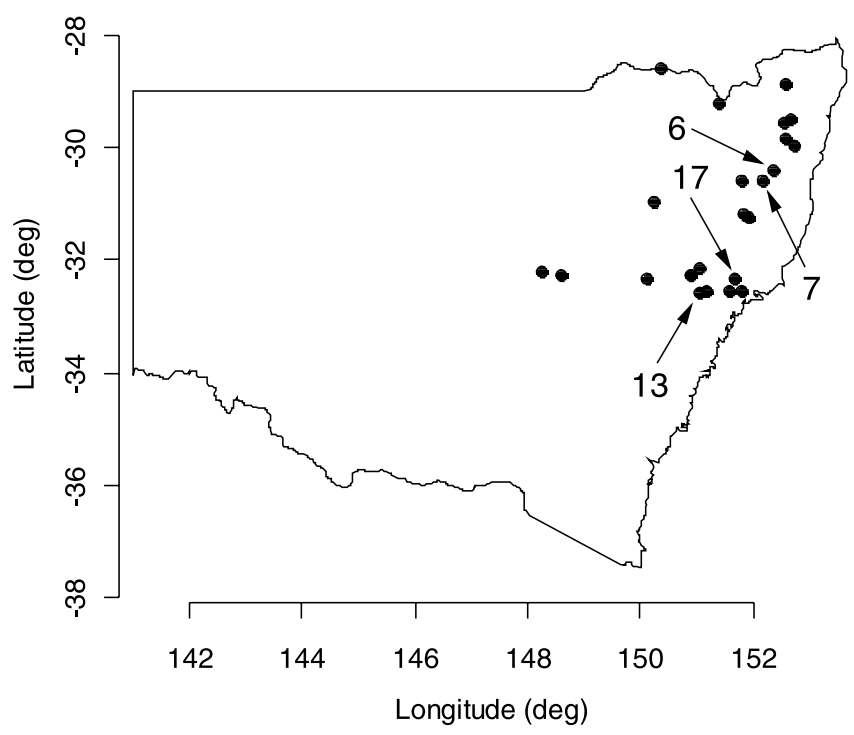

Figure 2. Location of the 24 case study sites within NSW, Australia. 
Table 2. Summary of Catchment Descriptor Variables and Record Length Used in Regional Regression Models

\begin{tabular}{lcccc}
\hline \multicolumn{1}{c}{ Variable } & Minimum & Maximum & Mean & Standard Deviation \\
\hline Log area $\left(\mathrm{km}^{2}\right)$ & 3.43 & 10.16 & 7.54 & 1.95 \\
Log IFD $(\mathrm{mm} / \mathrm{h})$ & 1.48 & 2.20 & 1.79 & 0.21 \\
Length (years) & 38 & 111 & 70.5 & 21.2 \\
\hline
\end{tabular}

[52] 2. The T-year flood is computed:

$$
q_{T}^{(j)}=\mu^{(j)}+K_{T}\left(\gamma^{(j)}\right) \sigma^{(j)}
$$

where $K_{T}$ is the frequency factor, with nonexceedance probability $1-1 / T$.

[53] 3. The likelihood function $p\left(S \mid \theta^{(j)}\right)$ is computed, which provides the unnormalized particle weight $w\left(\theta^{(j)}\right)$. If there are no site data available, then all particles have equal weight.

[54] 4. Steps $1-3$ are repeated for all $j=1, \ldots, N$ regional posterior samples.

[55] 5. The particle weights $P_{g}\left(\theta^{(j)}\right)$ are normalized using equation (28).

[56] 6. The $N$ values of the quantile $q_{T}$ are sorted, and the associated cumulative particle weights are computed.

[57] 7. Quantile probability limits are extracted using the cumulative particle weights.

[58] It is important to note that the above algorithm cannot be used if flood quantiles are regionalized (rather than distribution parameters). This is because the likelihood function in step 3 cannot be constructed given a quantile value.

\subsubsection{Results and Discussion}

[59] The regional flood data were analyzed using separate GLS regional regressions for each of the log Pearson III parameters using the block Metropolis sampler with $N=$ 55000 samples, where the first 5000 samples were discarded to allow the sampler to "warm up." Three catchment descriptors were used, namely constant, $\log$ area, and $\log$ IFD, where IFD is the 2-year, 12-h rainfall intensity [Canterford, 1987]. Table 2 presents summary statistics for the descriptors.
[60] Table 3 summarizes the posterior moments for the regression model parameters for several combinations of descriptors. Table 3 also reports the acceptance rate achieved for the regional error variances (using the uniform sampler; see section 3.3.2) and the diagnostic $p$ values from Kolmogorov-Smirnov (K-S) tests. The K-S tests [e.g., Ang and Tang, 2007, pp. 293-296] were performed using the $\mathrm{R}$ software package [ $R$ Development Core Team, 2008] to assess the adequacy of the regression models by checking the normality of their standardized model errors ("residuals") defined as

$$
R_{i}=\frac{z_{i}^{T} \beta-\hat{\theta}_{i}}{\sqrt{\sigma_{R}^{2}+\Sigma_{i i}}}
$$

where $R_{i}$ is the standardized residual for site $i$ and $\Sigma_{i i}$ is the appropriate diagonal element of the sampling covariance matrix. Appendix A summarizes the sampling covariance matrices for the LP III distribution.

[61] For the mean $(\mu)$ regression, the $\mathrm{m} 3$ model (using all 3 descriptors) is the preferred choice because it has, by far, the smallest regional model error variance, and the K-S test shows that the standardized model errors are consistent with the assumption of normality. Note that it could be argued that the $\mathrm{m} 2$ model would be a better choice because none of its coefficients contain zero in their $90 \%$ probability limits, while the constant coefficient for the m3 model does. However, this neglects the fact that the $\mathrm{m} 3$ model has a vastly superior expected regional error variance compared to $\mathrm{m} 2$; moreover, it seems unreasonable to exclude the constant coefficient in the regression model. For the standard deviation $(\sigma)$ regression, only two models (s1 and s2a) have coefficients that do not contain zero in their $90 \%$ probability limits, with model s2a being preferred because it has a smaller regional error variance. For the skewness $(\gamma)$ regression, the obvious choice would be the g1 model because it has the smallest regional error variance. However, it is excluded because its model errors are borderline normal. As a result, the g2 model was chosen even though the $90 \%$ probability intervals include zero.

[62] The posterior mean of $\sigma_{R}^{2}$ for regional skewness $\gamma$ is of particular interest. Noting that the asymptotic variance of

Table 3. Results of the Regional GLS Regression Analyses for the Mean, Standard Deviation, and Skewness Parameters of the LP III

\begin{tabular}{|c|c|c|c|c|c|c|}
\hline \multirow[b]{2}{*}{ Model } & \multicolumn{4}{|c|}{ Posterior Mean } & \multirow{2}{*}{$\begin{array}{c}\text { Uniform Sampler } \\
\text { Acceptance } \\
\text { Rate }(\%)\end{array}$} & \multirow{2}{*}{$\begin{array}{c}\text { K-S } p \text { Value } \\
\text { for Standardized } \\
\text { Residuals }\end{array}$} \\
\hline & Constant & Log Area & Log IFD & $\sigma_{R}^{2}$ & & \\
\hline $\mathrm{m} 1$ & $10.0544(0.2783)$ & & & $1.7250(0.5434)$ & 32.4 & 0.6793 \\
\hline $\mathrm{m} 2$ & $6.1522(0.6825)$ & $0.5176(0.0868)$ & & $0.6386(0.2111)$ & 37.5 & 0.3601 \\
\hline $\mathrm{m} 2 \mathrm{a}$ & $8.8098(2.3779)$ & & $0.6931(1.3144)^{\mathrm{a}}$ & $1.7878(0.5864)$ & 32.3 & 0.7082 \\
\hline $\mathrm{m} 3$ & $-1.1951(1.1469)^{\mathrm{a}}$ & $0.6786(0.0537)$ & $3.4089(0.4991)$ & $0.1808(0.0597)$ & 44.0 & 0.8922 \\
\hline s1 & $1.1420(0.0624)$ & & & $0.0543(0.0187)$ & 44.7 & 0.2219 \\
\hline s2 & $1.0117(0.2253)$ & $0.0167(0.0281)^{\mathrm{a}}$ & & $0.0563(0.0197)$ & 44.2 & 0.2498 \\
\hline $\mathrm{s} 2 \mathrm{a}$ & $2.1913(0.4201)$ & & $-0.5905(0.2310)$ & $0.0411(0.0150)$ & 44.9 & 0.5089 \\
\hline s3 & $2.3928(0.5985)$ & $-0.0137(0.0281)^{\mathrm{a}}$ & $-0.6430(0.2596)$ & $0.0425(0.0157)$ & 44.5 & 0.3242 \\
\hline g1 & $-0.2706(0.0912)$ & & & $0.0016(0.0053)$ & 2.5 & 0.0689 \\
\hline g2 & $0.0769(0.3041)^{\mathrm{a}}$ & $-0.0418(0.0350)^{\mathrm{a}}$ & & $0.0029(0.0071)$ & 5.3 & 0.3411 \\
\hline g2a & $-0.6245(0.6093)^{\mathrm{a}}$ & & $0.2025(0.3457)^{\mathrm{a}}$ & $0.0019(0.0059)$ & 3.0 & 0.2737 \\
\hline g3 & $0.0604(0.9035)^{\mathrm{a}}$ & $-0.0415(0.0398)^{\mathrm{a}}$ & $0.0077(0.3973)^{\mathrm{a}}$ & $0.0024(0.0067)$ & 4.3 & 0.3503 \\
\hline
\end{tabular}
Distribution

${ }^{\text {a }}$ Zero is contained in the $90 \%$ probability interval. Standard deviation is given in parentheses. 
Table 4. Correlation Between Standardized Regional Model Residuals and Associated $p$ Values From Significance Tests

\begin{tabular}{cccc}
\hline Model 1 & Model 2 & Correlation & $p$ Value \\
\hline $\mathrm{m} 3$ & $\mathrm{~s} 2 \mathrm{a}$ & -0.272 & 0.198 \\
$\mathrm{~m} 3$ & $\mathrm{~g} 2$ & 0.221 & 0.299 \\
$\mathrm{~s} 2 \mathrm{a}$ & $\mathrm{g} 2$ & 0.063 & 0.769 \\
\hline
\end{tabular}

sample skew is $6 / n, \sigma_{R}^{2}$ equal to 0.0029 has an equivalent sample size of 2069 years. This arises because virtually all of the variation of the sample skew about the regional regression relationship g2 can be attributed to sampling error. Nonetheless, note that the posterior standard deviation on $\sigma_{R}^{2}$ is 0.0071 , which indicates there is very large uncertainty about the true value of $\sigma_{R}^{2}$. Importantly, step 1 in the quantile inference procedure of section 5.2.2 properly accounts for this uncertainty.

[63] The achieved uniform sampler acceptance rates were quite good for the mean and standard deviation regressions; Gelman et al. [1995, p. 335] states that, for normal posteriors, the optimal acceptance rate is about 0.4. The skewness acceptance rates were poorer and due to the fact that sampling error dominated model error. The skewness acceptance rates of $2-5 \%$ are at the lower end of the desirable range; if the rates had dropped much further,
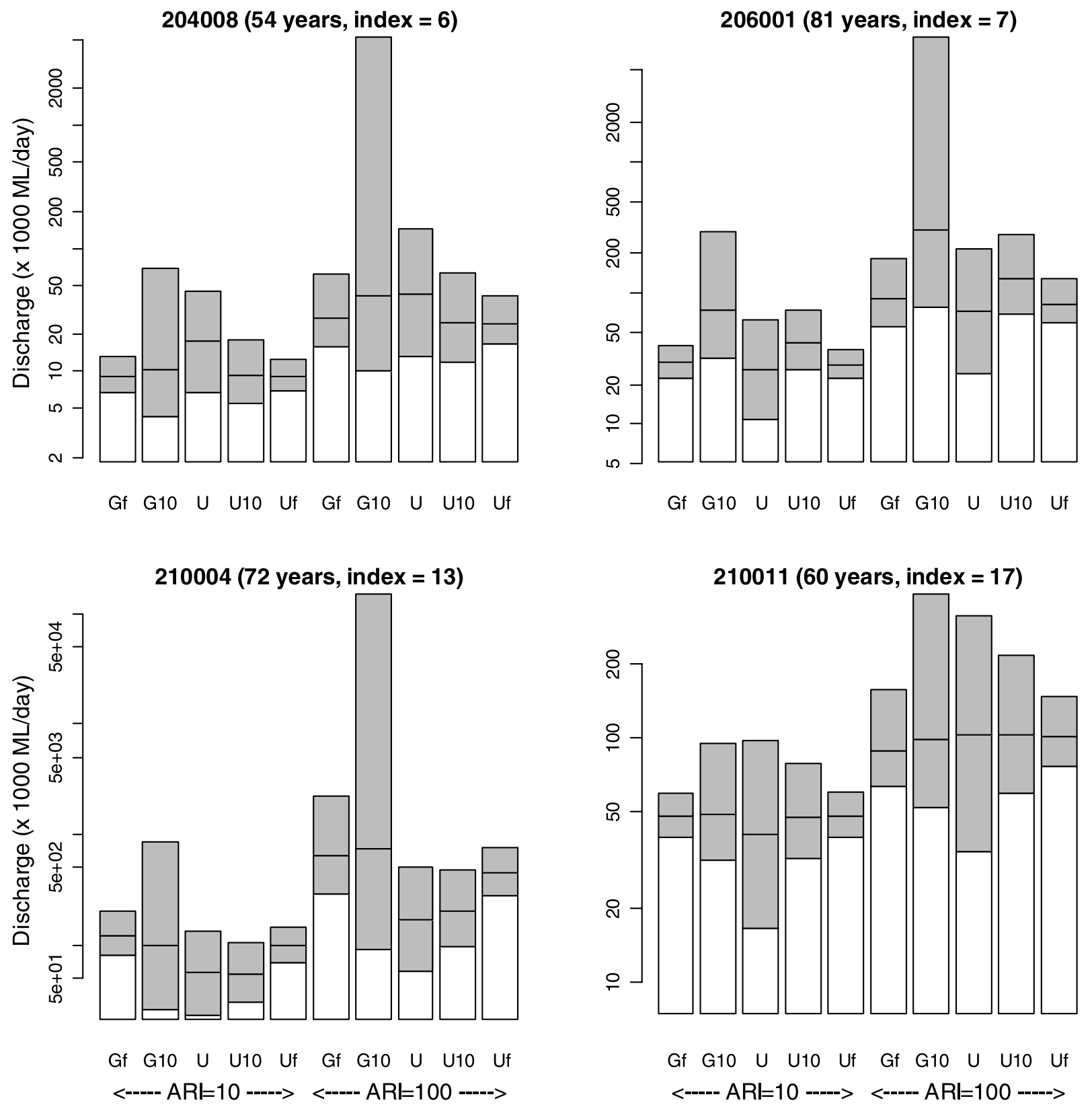

Figure 3. Posterior distributions of 10- and 100-year flood quantiles with boxes showing posterior median and $90 \%$ probability limits. Gf is the full (at-site) gauged record, G10 uses 10 years of gauged data, $\mathrm{U}$ is the regional distribution only ("ungauged"), U10 is ungauged data pooled with 10 years of gauged data, and Uf is ungauged data pooled with the full gauged record. 
action would have been taken to improve the rates, such as decreasing the value of $K$ (equation (12)).

[64] A key assumption is that the regional model errors for $\mu, \sigma$, and $\gamma$ are statistically independent. This can be tested approximately by analyzing the correlations between the standardized model residuals. Table 4 shows that the correlations between the models are small, with a maximum of 0.27 . In addition, Table 4 reports $p$ values testing the hypothesis that the correlation is zero (the tests were performed using the function "cor.test" in the R software package). The smallest $p$ value is 0.198 , which indicates that there is insufficient evidence to reject the null hypothesis that the correlation is zero for any model pair.

[65] The regional pooling procedure is illustrated for several scenarios. Two gauged data scenarios are considered: Gf, the full gauged record; and G10, 10 years of data randomly sampled from the full gauged record. These scenarios are used to determine the improvements, if any, that may be gained from pooling at-site information with regional information. Figure 3 shows the 10- and 100-year flood quantile distributions for 4 sites. For each site and flood quantile, five distributions are displayed with the following labels: Gf, at-site distribution for the full gauged record; G10, at-site distribution for the randomly sampled 10-year gauged record; U, distribution using regional model only (this is the ungauged catchment case); U10, pooled distribution using regional model and 10-year gauged record; Uf, pooled distribution using regional model and full gauged record.

[66] The regional quantiles $U$ exhibit less uncertainty than the G10 quantiles for 3 of the 4 sites, particularly for the 100-year quantile. This illustrates the not insignificant information content of the regional model. Most likely this benefit comes from the skewness regional model. With only 10 years of gauged data, the skewness sample errors are large and can induce very large uncertainty in extreme quantiles. This is a timely reminder of the motivation for using regional skewness in Bulletin 17B.

[67] Pooling 10 years of gauged flows with regional information brings about considerable reductions in quantile uncertainty. In some cases, the uncertainty is similar to that for the full gauged record. Not unexpectedly, pooling the full gauged record with regional information reduces uncertainty below that of the full gauged record.

[68] Site 206001 is interesting because the shortened 10year record appears quite different to the full 81-year gauged record; by chance the shortened record consists of higher-than-average flow years, leading to the shortened flood distribution having an upward bias. The pooled distribution U10 is quite consistent with the full gauged distribution Gf. This illustrates that the use of the regional pooling procedure can help guard against bias in the resultant flood frequency distribution if a short, gauged record is unrepresentative of the long-run flood risk. However, it should be noted that there is potential for the regional model to misspecify the flood distribution at outlier sites.

\section{Conclusion}

[69] This study has presented a general Bayesian approach for inferring the GLS regional regression model and for pooling with any available site information to obtain the most accurate flood quantiles at a particular site.
The approach uses a robust block Metropolis scheme to sample the posterior distribution of the GLS regression model parameters. Moreover, it uses a simple but general procedure, based on importance sampling, to pool any kind of site information with regional information. The benefit of this approach is that all available information is fully exploited and uncertainty is rigorously quantified as well as minimized.

[70] Two case studies were used to illustrate important characteristics of the regionalization problem. The first, a synthetic case study, illustrated the complex nature of the posterior distribution for the regional model error variance with its shape dependent on the degree that sampling error dominates the total error in the regional GLS model. It highlights the need for a full Bayesian analysis to properly account for uncertainty. The second case study, involving 24 sites from the east coast of Australia, demonstrated the significant benefit of pooling site and regional information and the quantification of uncertainty.

[71] Although the Bayesian method was illustrated for the log Pearson III distribution, it can be applied to other flood distributions (using the appropriate sampling covariance matrices) or to flood quantiles directly (although pooling with site information is not possible). The algorithms have been implemented in the Fortran 95 programming language, with executable files and documentation freely available for research purposes from the first author, on request.

\section{Appendix A: Sampling Covariance Matrices for the LP III Distribution}

[72] For completeness, this appendix describes the sampling covariance matrices associated with method-ofmoments estimators for the mean, standard deviation, and skewness of the log Pearson III distributions for the case when gauged data is the only flood information available.

[73] Suppose that at each of $n$ sites, there is available a $n_{i}$-year flow record $\left\{q_{i, 1}, \ldots, q_{i, n_{i}}\right\}$. These observations are assumed to be temporally independent samples of annual maximum flows (or of similar quantities, or some transformation thereof). Concurrent flows at different sites may be spatially correlated. Working with the (natural) logarithms of the flows, the sample mean $\left(\bar{q}_{i}\right)$, standard deviation $\left(s_{i}\right)$, and skewness $\left(g_{i}\right)$ at each site are estimated as

$$
\begin{aligned}
\bar{q}_{i} & =\frac{1}{n_{i}} \sum_{k=1}^{n_{i}} q_{i, k} \\
s_{i}^{2} & =\frac{1}{n_{i}-1} \sum_{k=1}^{n_{i}}\left(q_{i, k}-\bar{q}_{i}\right)^{2} \\
g_{i} & =\frac{n_{i}}{\left(n_{i}-1\right)\left(n_{i}-2\right) s_{i}^{3}} \sum_{k=1}^{n_{i}}\left(q_{i, k}-\bar{q}_{i}\right)^{3}
\end{aligned}
$$

and the cross correlation between concurrent flows at two sites $i$ and $j$, separated by distance $d_{i j}$, is estimated using an (exponential type) distance-decay function:

$$
\rho_{i j}=\exp \left[-\left(d_{i j} / A\right)^{B}\right]
$$

where the parameters $A$ and $B$ require estimation. Importantly, equation (A2) provides a smoothed estimate of cross correlation. 
[74] Following Stedinger and Tasker [1985, 1986b], the sampling covariance matrices for the sample mean $\left(\theta=\bar{q}_{i}\right)$ of the lognormal and log Pearson III distributions are identical and are given by

$$
\begin{gathered}
\Sigma(\bar{q})_{i j}=\sigma_{i}^{2} / n_{i} \quad \text { for } i=j \\
\Sigma(\bar{q})_{i j}=\rho_{i j} m_{i j} \sigma_{i} \sigma_{j} /\left(n_{i} n_{j}\right) \quad \text { for } i \neq j
\end{gathered}
$$

where the (unbiased) sample standard deviation $s_{i}$ is used in place of the population standard deviation $\sigma_{i}$, the smoothed cross correlation between concurrent flows is used for $\rho_{i j}$, and $m_{i j}$ is the number of concurrent flows between sites $i$ and $j$.

[75] The sampling covariance for the sample standard deviation $\left(\theta=s_{i}\right)$ is

$$
\begin{gathered}
\Sigma(s)_{i j}=0.5\left(1+0.75 \gamma_{i}^{2}\right) \sigma_{i}^{2} / n_{i} \quad \text { for } i=j \\
\Sigma(s)_{i j}=0.5\left(\rho_{i j}+0.75 \gamma_{i} \gamma_{j}\right) \rho_{i j} m_{i j} \sigma_{i} \sigma_{j} /\left(n_{i} n_{j}\right) \quad \text { for } i \neq j
\end{gathered}
$$

The procedure for the standard deviation of Pearson III variates requires care to avoid correlation between the residuals and the estimated standard deviations [Griffis and Stedinger, 2007b]: (1) $\rho_{i j}$ is estimated as a function of distance between sites $i$ and $j$ (see equation (A2)); (2) regional (average) skew is used in place of the population skew (i.e., regional average skew $=g_{\mathrm{av}}=\gamma_{i}=\gamma_{j}$ ); and (3) the standard deviations $\sigma_{i}$ and $\sigma_{j}$ are estimated using an independent OLS regression using the appropriate descriptors $Z$ for the standard deviation.

[76] The sampling covariance matrix for the sample skewness $\left(\theta=g_{i}\right)$ is [Reis et al., 2005; Griffis and Stedinger, 2007b]

$$
\begin{gathered}
\Sigma(g)_{i j}=\operatorname{Var}\left(g_{i}\right) \quad \text { for } i=j \\
\Sigma(g)_{i j}=\rho\left(g_{i}, g_{j}\right) \sqrt{\operatorname{Var}\left(g_{i}\right) \operatorname{Var}\left(g_{j}\right)} \quad \text { for } i \neq j
\end{gathered}
$$

where

$$
\begin{aligned}
\operatorname{Var}\left(g_{i}\right)= & {\left[6 / n_{i}+a\left(n_{i}\right)\right]\left\{1+\left[9 / 6+b\left(n_{i}\right)\right] \gamma_{i}^{2}\right.} \\
& \left.+\left[15 / 48+c\left(n_{i}\right)\right] \gamma_{i}^{4}\right\}
\end{aligned}
$$

and the small sample corrections are given by

$$
\begin{aligned}
& a(n)=-17.75 / n^{2}+50.06 / n^{3} \\
& b(n)=3.92 / n^{0.3}-31.1 / n^{0.6}+34.86 / n^{0.9} \\
& c(n)=-7.31 / n^{0.59}+45.9 / n^{1.18}-86.5 / n^{1.77}
\end{aligned}
$$

The skewness correlation is approximated by [Martins and Stedinger, 2002]

$$
\rho\left(g_{i}, g_{j}\right)=\operatorname{Sign}\left(\rho_{i j}\right) c f_{i j}\left|\rho_{i j}\right|^{\nu}
$$

where $\operatorname{Sign}\left(\rho_{i j}\right)$ is \pm 1 , depending on the sign of $\rho_{i j}$, and the correction factor is

$$
c f_{i j}=m_{i j} / \sqrt{n_{i} n_{j}}
$$

and the exponent $\nu$, tabulated by Martins and Stedinger [2002] for $|\gamma| \leq 1$, depends on the regional skewness and has a typical range of $2.8 \leq \nu \leq 3.3$.

[77] Acknowledgments. This research was partly funded by an Australian Research Council Discovery Grant. The helpful comments of John England, two anonymous reviewers, and the associate editor are gratefully acknowledged.

\section{References}

Ang, A. H.-S., and W. H. Tang (2007), Probability Concepts in Engineering: Emphasis on Applications in Civil and Environmental Engineering, 2nd ed., 406 pp., John Wiley, New York.

Canterford, R. P. (Ed.) (1987), Australian Rainfall and Runoff: A Guide to Flood Estimation, vol. 2, Inst. of Eng. Aust., Barton, ACT, Australia.

Chib, S., and E. Greenberg (1995), Understanding the Metropolis-Hastings algorithm, Am. Stat., 49(4), 327-335.

Gelman, A., J. B. Carlin, H. S. Stern, and D. B. Rubin (1995), Bayesian Data Analysis, 526 pp., Chapman and Hall, London.

Geman, S., and D. Geman (1984), Stochastic relaxation, Gibbs distributions, and the Bayesian restoration of images, IEEE Trans. Pattern Anal. Mach. Intell., 6(6), 721-741.

Griffis, V. W., and J. R. Stedinger (2007a), Evolution of flood frequency analysis with Bulletin 17, J. Hydrol. Eng., 12(3), 283-297, doi:10.1061/ (ASCE)1084-0699(2007)12:3(283).

Griffis, V. W., and J. R. Stedinger (2007b), The use of GLS regression in regional hydrologic analyses, J. Hydrol., 344(1-2), 82-95, doi:10.1016/ j.jhydrol.2007.06.023.

Hastings, W. K. (1970), Monte Carlo sampling methods using Markov chains and their applications, Biometrika, 57(1), 97-109.

Interagency Advisory Committee on Water Data (1982), Guidelines For Determining Flood Flow Frequency, Bull. 17B, Hydrol. Subcomm., Washington, D. C.

Kuczera, G. (1983), Effect of sampling uncertainty and spatial correlation on an empirical Bayes procedure for combining site and regional information, J. Hydrol., 65(4), 373-398, doi:10.1016/0022-1694(83)90088-4

Kuczera, G. (1999), Comprehensive at-site flood frequency analysis using Monte Carlo Bayesian inference, Water Resour. Res., 35(5), 1551-1558.

Marshall, L., D. Nott, and A. Sharma (2004), A comparative study of Markov chain Monte Carlo methods for conceptual rainfall-runoff modeling, Water Resour. Res., 40, W02501, doi:10.1029/2003WR002378.

Martins, E. S., and J. R. Stedinger (2002), Cross correlations among estimators of shape, Water Resour. Res., 38(11), 1252, doi:10.1029/ 2002WR001589.

Metropolis, N., A. W. Rosenbluth, M. N. Rosenbluth, A. H. Teller, and E. Teller (1953), Equation of state calculations by fast computing machines, J. Chem. Phys., 21(6), 1087-1092, doi:10.1063/1.1699114.

Micevski, T., G. Kuczera, and S. W. Franks (2005), Flood frequency censoring errors associated with daily-read flood observations, Water Resour. Res., 41, W07002, doi:10.1029/2004WR003881.

R Development Core Team (2008), R: A Language and Environment for Statistical Computing, R Found. for Stat. Comput., Vienna.

Reis, D. S., Jr., J. R. Stedinger, and E. S. Martins (2005), Bayesian generalized least squares regression with application to $\log$ Pearson type 3 regional skew estimation, Water Resour. Res., 41, W10419, doi:10.1029/ 2004WR003445.

Robert, C. P., and G. Casella (2004), Monte Carlo Statistical Methods, 2nd ed., Springer, New York.

Stedinger, J. R., and T. A. Cohn (1986), Flood frequency analysis with historical and paleoflood information, Water Resour. Res., 22(5), $785-$ 793.

Stedinger, J. R., and G. D. Tasker (1985), Regional hydrologic analysis: 1. Ordinary, weighted and generalized least squares compared, Water Resour. Res., 21(9), 1421-1432.

Stedinger, J. R., and G. D. Tasker (1986a), Correction to "Regional hydrologic analysis: 1. Ordinary, weighted and generalized least squares compared", Water Resour. Res., 22(5), 844. 
Stedinger, J. R., and G. D. Tasker (1986b), Regional hydrologic analysis: 2. Model-error estimators, estimation of sigma, and log-Pearson type 3 distributions, Water Resour. Res., 22(10), 1487-1499.

Tasker, G. D., and J. R. Stedinger (1989), An operational GLS model for hydrologic regression, J. Hydrol., 111(1-4), 361-375.

Tasker, G. D., J. H. Eychaner, and J. R. Stedinger (1987), Application of generalized least squares in regional hydrologic regression analysis, U.S. Geol. Surv. Water Supply Pap., 2310, 107-115.
Theil, H. (1971), Principles of Econometrics, 736 pp., John Wiley, New York.

G. Kuczera and T. Micevski, Discipline of Civil, Surveying, and Environmental Engineering, University of Newcastle, Callaghan, NSW 2308, Australia. (george.kuczera@newcastle.edu.au; tom.micevski@newcastle. edu.au) 